\title{
Shorter Leukocyte Telomere Length Is Associated With Atrial Remodeling and Predicts Recurrence in Younger Patients With Paroxysmal Atrial Fibrillation After Radiofrequency Ablation
}

\author{
Kuo-Li Pan, MD; Ya-Wen Hsiao, PhD; Yenn-Jiang Lin, MD; Li-Wei Lo, MD; \\ Yu-Feng Hu, MD; Fa-Po Chung, MD; Yung-Nan Tsai; Tze-Fan Chao, MD; \\ Jo-Nan Liao, MD; Chin-Yu Lin, MD; Shih-Jie Jhuo, MD; Chung-Hsing Lin, MD; \\ Allamsetty Suresh, MD; Rohit Walia, MD; Abigail Louise D. Te, MD; Shinya Yamada, MD; \\ Yao-Ting Chang, MD; Shih-Lin Chang, MD; Shih-Ann Chen, MD
}

\begin{abstract}
Background: Telomere length is a biologic aging marker. This study investigated leukocyte telomere length (LTL) as a new biomarker to predict recurrence after paroxysmal atrial fibrillation (PAF) ablation.
\end{abstract}

\begin{abstract}
Methods and Results: A total of 131 participants (26 healthy individuals and 105 symptomatic PAF patients) were enrolled. PAF patients (54.1 \pm 10.8 years) who received catheter ablation therapy were divided into 2 groups: recurrent $A F(n=25)$ and no recurrent AF after catheter ablation $(n=80)$. Peripheral blood mononuclear cells were collected from all subjects to measure LTL. Under 50 years old, $L T L$ in healthy individuals $(n=17)$ was longer than in PAF patients $(n=31 ; 7.34 \pm 0.58 \mathrm{kbp}$ vs. $6.44 \pm 0.91 \mathrm{kbp}, P=0.01)$. In $P A F$ patients, $L T L$ was positively correlated with left atrial bipolar voltage $(R=0.497, P<0.001)$, and negatively correlated with biatrial scar area $(R=-0.570, P<0.001)$ and left atrial diameter $(R=-0.214, P=0.028)$. $L T L$ was shorter in the patients with recurrent $A F$ than in those without recurrent $A F$ after catheter ablation $(5.68 \pm 0.82 \mathrm{kbp}$ vs. $6.66 \pm 0.71 \mathrm{kbp} ; \mathrm{P}<0.001)$. On receiver operating characteristic curve analysis, LTL cut-off $<6.14 \mathrm{kbp}$ had a specificity of 0.68 and sensitivity of 0.79 to predict recurrent AF after catheter ablation.
\end{abstract}

Conclusions: Young PAF patients ( $\leq 50$ years) had shorter LTL. Shorter LTL was associated with a degenerative atrial substrate and recurrence after catheter ablation in younger PAF patients.

Key Words: Atrial fibrillation; Catheter ablation; Leukocyte telomere length

A trial fibrillation (AF) is the most common cardiac arrhythmia leading to increased mortality, risk of stroke, heart failure and hospitalization. ${ }^{1}$ Approximately $70 \%$ of AF patients are between 65 and 85 years of age; the incidence of AF increases with age, and the median AF patient age is approximately 75 years. 2,3 In recent years, radiofrequency (RF) catheter ablation of pulmonary veins (PV) has become a standard and effective therapy for symp-

\section{Editorial p1439}

tomatic and drug-refractory paroxysmal AF (PAF). ${ }^{4}$ Clinically, patients with high $\mathrm{CHADS}_{2}$ score, ${ }^{5}$ old age $(\geq 65$ years), ${ }^{6}$ and presence of associated cardiovascular disease and poor left atrial (LA) substrate ${ }^{7}$ have a high incidence of recurrent AF after RF catheter ablation. Aging is also cor-

Received August 24, 2018; revised manuscript received April 4, 2019; accepted April 9, 2019; J-STAGE Advance Publication released online May 23, 2019 Time for primary review: 46 days

Division of Cardiology, Chang Gung Memorial Hospital, Chiayi (K.-L.P.); School of Traditional Chinese Medicine, College of Medicine, Chang Gung University, Taoyuan (K.-L.P.); Division of Cardiology, Department of Medicine, Taipei Veterans General Hospital, Taipei (Y.-W.H., Y.-J.L., L.-W.L., Y.-F.H., F.-P.C., Y.-N.T., T.-F.C., J.-N.L., C.-Y.L., Y.-T.C., S.-L.C., S.-A.C.); School of Medicine, Faculty of Medicine, National Yang-Ming University, Taipei (Y.-J.L., L.-W.L., Y.-F.H., F.-P.C., Y.-N.T., T.-F.C., J.-N.L., C.-Y.L., Y.-T.C., S.-L.C., S.-A.C.); Division of Cardiology, Kaohsiung Medical University Hospital, Kaohsiung (S.-J.J.); Division of Cardiology, Department of Internal Medicine, Shuang Ho Hospital, Taipei Medical University, Taipei (C.-H.L.), Taiwan; Department of Cardiology, Nizam's Institute of Medical Sciences, Hyderabad (A.S.); Division of Cardiology, Medanta Heart Institute, Medanta The Medicity, Gurgaon, Haryana (R.W.), India; Heart Institute, St. Luke's Medical Center, Quezon City (A.L.D.T.), The Philippines; and Department of Cardiology and Hematology, Fukushima Medical University, Hikarigaoka, Fukushima (S.Y.), Japan

The first two authors contributed equally to this work (K.-L.P., Y.-W.H.).

Mailing address: Shih-Lin Chang, MD, PhD, Heart Rhythm Center, Division of Cardiology, Department of Medicine, Taipei Veterans General Hospital, 201 Section 2, Shih-Pai Road, Taipei, Taiwan. E-mail: slchang4@vghtpe.gov.tw

ISSN-1346-9843 All rights are reserved to the Japanese Circulation Society. For permissions, please e-mail: cj@j-circ.or.jp 
related with structural and electric remodeling, including dilated LA volume and increased areas of LA low voltage, which may be responsible for the raised propensity to AF and higher recurrence of AF after ablation., ${ }^{6,8}$ Some biomarkers are related to AF, such as inflammatory, oxidative stress markers. ${ }^{9}$ A biomarker that is causally associated with AF genesis and which predicts the outcome after RF catheter ablation, however, remains to be identified.

Telomeres are protein-nucleotide complexes containing thousands of repetitive DNA sequences (TTAGGG) located at both ends of each chromosome to protect the chromosomal DNA, reduce the risk of apoptosis and to stabilize the DNA complex. ${ }^{10}$ In each cell division, telomere repetitive sequences will become shorter, eventually reaching a critical threshold leading to cellular senescence and death. ${ }^{11}$ Therefore, telomere length is thought of as a biologic aging marker representing cellular age and is associated with aging-related disease. Shorter leukocyte telomere length (LTL) has been shown to be associated with hypertension, diabetes mellitus (DM), coronary artery disease and heart failure. ${ }^{\mathbf{1 2}-15}$ Because aging has an impact on post-catheter ablation recurrent AF and LA remodeling, we hypothesize that LTL might be a biomarker for prediction of recurrent AF after ablation. ${ }^{6}$ This study therefore investigated the impact of LTL on atrial substrate remodeling and whether it can predict the occurrence of AF in a selected population as well as recurrence after ablation in patients with PAF.

\section{Methods}

\section{Subjects and Study Design}

To study the association between LTL and incident PAF, 131 participants consisting of 26 healthy individuals (male/ female, 14/12; mean age, $43.42 \pm 10.23$ years) and 105 symptomatic and drug-refractory PAF patients (male/female, 72/33; mean age, $54.07 \pm 10.8$ years) who received catheter ablation were consecutively enrolled in this retrospective study between August 2014 and June 2016 at Taipei Veterans General Hospital (a tertiary center). Furthermore, PAF patients were divided into 2 groups: post-catheter ablation recurrent $\mathrm{AF}(\mathrm{n}=25)$, and no recurrent $\mathrm{AF}$ after catheter ablation $(n=106)$. Exclusion criteria consisted of left ventricular (LV) systolic dysfunction, moderate-severe valvular heart disease, congenital heart disease, or history of previous cardiac surgery. This study was approved by the Taipei Veterans General Hospital Institutional Review Board, and informed consent was obtained from all patients who participated in this study.

\section{Baseline Data}

Patient clinical baseline characteristics including age, sex, height, body weight, body mass index (BMI), body surface area (BSA), creatinine, serum triglyceride, serum cholesterol, echocardiography data and the absence or presence of hypertension, DM, and hypercholesterolemia were obtained from medical records before RF catheter ablation for PAF.

\section{RF Catheter Ablation}

The details have been described previously. ${ }^{\mathbf{1 6}, 17}$ In brief, all anti-arrhythmic drugs except for amiodarone were discontinued for at least 5 half-lives, and transesophageal echocardiography was also performed to exclude LA thrombus in each patient before electrophysiological study (EPS) and RF catheter ablation. All of the patients underwent EPS and RF catheter ablation in a fasting, non-sedated state. After the trans-septal access was created, heparin was used to avoid thrombus formation with a targeted activated clotting time $250-300 \mathrm{~s}$. The $3-\mathrm{D}$ geometry of the LA was then captured by moving a mapping catheter (Spiral SC, AF Division, St. Jude Medical, MN, USA) over the endocardial surface of the LA and 4 PV, using the NavX contact mapping system (St. Jude Medical). The ostia of the 4 PV were also identified on fluoroscopy and marked on the 3-D map of the LA. PV isolation was then carried out with circumferential ablation around the ostium of the PV using an irrigated-tip 4-mm ablation catheter (Cool Path ${ }^{\mathrm{TM}}$ Irrigated Ablation Catheter, St. Jude Medical). RF energy was applied continuously while repositioning the catheter tip every $40 \mathrm{~s}$ with a target temperature of $35-40^{\circ} \mathrm{C}$ and maximum power $20-25 \mathrm{~W}$ at the posterior LA wall, and 25-30 W at the anterior LA wall in the power control mode. After completion of the circumferential lesion set, the ipsilateral superior and inferior PV were mapped carefully on circular catheter recording during sinus rhythm (SR) or coronary sinus pacing. Supplementary ablation applications were applied along the circumferential lines close to the earliest ipsilateral PV spikes. Absence of any PV potential or presence of dissociated PV activity was the endpoint of PV isolation.

\section{Right and Left Atrium Voltage Mapping}

The details have been described previously. ${ }^{7}$ In brief, before PV isolation, the irrigated-tip 4-mm ablation catheter was used to collect the right atrial (RA) and LA bipolar local voltages while the catheter was in contact with the atrial wall as it was moved through the RA and LA during SR under the NavX contact mapping system. For the patients with the rhythm of AF before PV isolation, voltage mapping was performed after termination of AF by catheter ablation or cardioversion after PV isolation. The signal from the roving catheter was used to build a sequential map. After completion of the sequential map, the bipolar voltage mapping points were analyzed using offline software to determine the mean voltage, and to measure the areas of RA and LA scar zone. "Scar" was defined as an absence of any voltage or a bipolar voltage amplitude $<0.05 \mathrm{mV}$. Scar area index was defined as scar surface area/ total surface area of the atrium.

\section{LTL Measurement}

Blood was obtained before RF catheter ablation. Telomere length was assessed on TeloTAGGG Telomere Length Assay, which relies on the absence of certain restriction enzyme recognition sites in the telomeric 5'-TTAGGG-3' tandem repeat sequences. DNA was extracted from the peripheral blood mononuclear cells using a standard commercially available methodology. After digestion of the overall genomic DNA by an optimized mixture of frequently cutting restriction enzymes, which principally left telomeric sequences intact, the terminal restriction fragments (TRF) were separated on gel electrophoresis and transferred onto polyvinylidene difluoride membranes. Using chemiluminescence detection for TRF analysis, the membranes were then blocked for $30 \mathrm{~min}$ and hybridized with telomere-specific digoxigenin (DIG)-labeled hybridization probe, and then incubated with anti-DIG-alkaline phosphatase for $1 \mathrm{~h}$. Finally, the membranes were exposed on X-ray film for $16 \mathrm{~min}$. Mean TRF length was defined 


\begin{tabular}{|c|c|c|c|c|c|c|c|c|c|}
\hline & \multirow[b]{2}{*}{$\begin{array}{l}\text { Control } \\
(n=26)\end{array}$} & \multirow[b]{2}{*}{$\begin{array}{c}\text { PAF } \\
(n=105)\end{array}$} & \multirow[b]{2}{*}{$\begin{array}{c}\mathrm{P}- \\
\text { value }\end{array}$} & \multicolumn{2}{|c|}{ After catheter ablation } & \multirow[b]{2}{*}{$\begin{array}{c}\mathrm{P} \text { - } \\
\text { value }\end{array}$} & \multicolumn{3}{|c|}{ Univariate } \\
\hline & & & & $\begin{array}{l}\text { Recurrence } \\
(n=25)\end{array}$ & $\begin{array}{c}\text { No } \\
\text { recurrence } \\
(n=80)\end{array}$ & & HR & $\begin{array}{c}\text { OR } \\
(95 \% \mathrm{Cl})\end{array}$ & $\begin{array}{c}\mathrm{P} \text { - } \\
\text { value }\end{array}$ \\
\hline Age (years) & $43.42 \pm 10.23$ & $54.07 \pm 10.80$ & $<0.001$ & $53.76 \pm 11.18$ & $54.16 \pm 10.75$ & 0.872 & 0.997 & $0.956-1.039$ & 0.870 \\
\hline Male & $14(53.8)$ & $72(68.6)$ & 0.157 & $16(64)$ & $56(70)$ & 0.573 & 0.762 & $0.296-1.963$ & 0.573 \\
\hline $\mathrm{BMI}\left(\mathrm{kg} / \mathrm{m}^{2}\right)$ & $26.18 \pm 4.07$ & $25.49 \pm 3.27$ & 0.359 & $25.81 \pm 3.69$ & $25.39 \pm 3.15$ & 0.580 & & & \\
\hline $\mathrm{BSA}\left(\mathrm{m}^{2}\right)$ & $1.92 \pm 0.29$ & $1.83 \pm 0.16$ & 0.13 & $1.841 \pm 0.18$ & $1.824 \pm 0.15$ & 0.624 & & & \\
\hline Creatinine (mg/dL) & $0.88 \pm 0.26$ & $0.95 \pm 0.20$ & 0.196 & $0.91 \pm 0.18$ & $0.97 \pm 21$ & 0.228 & & & \\
\hline Diabetes mellitus & $2(7.7)$ & $12(11.4)$ & 0.581 & $2(8.0)$ & $10(12.5)$ & 0.537 & & & \\
\hline Hypertension & 7 (26.9) & $43(41.0)$ & 0.187 & $12(48)$ & $31(38.8)$ & 0.412 & & & \\
\hline Hypercholesterolemia & $2(7.7)$ & $7(6.7)$ & 0.853 & $2(8.0)$ & $5(6.3)$ & 0.759 & & & \\
\hline $\mathrm{IL}-1$ (pg/mL) & $1.17 \pm 0.91$ & $4.80 \pm 0.87$ & $<0.001$ & $5.62 \pm 0.58$ & $4.55 \pm 0.79$ & $<0.001$ & & & \\
\hline IL-6 (pg/mL) & $12.38 \pm 6.17$ & $28.48 \pm 9.29$ & $<0.001$ & $36.87 \pm 11.42$ & $25.85 \pm 6.69$ & $<0.001$ & & & \\
\hline LTL (kbp) & $7.19 \pm 0.54$ & $6.40 \pm 0.84$ & $<0.001$ & $5.68 \pm 0.82$ & $6.66 \pm 0.71$ & $<0.001$ & 0.193 & $0.093-0.400$ & $<0.001$ \\
\hline $\operatorname{LVEF}(\%)$ & & & & $60.24 \pm 5.07$ & $60.48 \pm 6.01$ & 0.86 & 0.993 & $0.919-1.073$ & 0.858 \\
\hline LA diameter $(\mathrm{mm})$ & & & & $39.00 \pm 5.38$ & $34.94 \pm 5.36$ & 0.001 & 1.184 & $1.068-1.313$ & 0.001 \\
\hline LA voltage $(\mathrm{mV})$ & & & & $1.66 \pm 0.17$ & $1.90 \pm 0.22$ & $<0.001$ & 0.004 & $0.000-0.057$ & $<0.001$ \\
\hline Biatrial scar area index (\%) & & & & $3.76 \pm 1.18$ & $2.55 \pm 0.62$ & $<0.001$ & 4.361 & $2.339-8.131$ & $<0.001$ \\
\hline
\end{tabular}

Data given as mean $\pm \mathrm{SD}$ or $\mathrm{n}(\%)$. AF, atrial fibrillation; BMI, body mass index; BSA, body surface area; IL, interleukin; LA, left atrium; LTL, leukocyte telomere length; LVEF, left ventricular ejection fraction; PAF, paroxysmal AF.

according to the following formula:

$$
\overline{\mathrm{TRF}}=\frac{\sum\left(\mathrm{OD}_{\mathrm{i}}\right)}{\sum\left(\mathrm{OD}_{\mathrm{i}} / \mathrm{Li}_{\mathrm{i}}\right)}
$$

Where $\mathrm{OD}_{\mathrm{i}}$ is the chemiluminescent signal and $\mathrm{Li}_{\mathrm{i}}$ is the length of the TRF at position $i$. The calculation takes into account the higher signal intensity from larger TRF due to multiple hybridizations of the telomere-specific hybridization probe.

\section{Enzyme-Linked Immunosorbent Assay (ELISA)}

Because the pro-inflammatory markers interleukin (IL)-1 and IL-6 were reported to be associated with senescence and LTL shortening, ${ }^{18,19}$ participant plasma was collected from peripheral blood, and IL-1 and IL- 6 were measured on ELISA, following the manufacturer's instructions (SigmaAldrich). All samples were measured in duplicate.

\section{Post-Ablation Follow-up}

Patients underwent follow-up ( 2 weeks after the catheter ablation, then every 3 months thereafter) at the cardiology clinic for 1 year. Anti-arrhythmic drugs were prescribed to prevent any early recurrence of AF in the blanking period, defined as $<3$ months after ablation, and the patients with tachycardia other than AF were closely monitored in the outpatient clinics. During each 3-month follow-up, 24-h Holter monitoring and/or cardiac event recording with a recording duration of 1 week were performed. Recurrent AF was defined as an episode of AF lasting $>1$ min on 24-h Holter or cardiac event recording. If more than 1 episode of atrial flutter, recurrent symptomatic AF, or atrial tachycardia was documented, the patients were encouraged to receive another ablation, or anti-arrhythmic drugs were prescribed to control the recurrent atrial arrhythmias. Long-term efficacy was assessed in all patients on the basis of the clinical symptoms, resting surface 12-lead electrocardiogram (ECG), and 24-h Holter monitor, and/or 1-week cardiac event recordings.

\section{Statistical Analysis}

Statistical analysis was performed using SPSS 18.0 (SPSS, Chicago, IL, USA). Continuous data are presented as mean \pm SD and dichotomous data as absolute values with percentage. Comparison of continuous variables between groups was performed using Student's t-test (2-tailed) for parametric data and Mann-Whitney U-test for non-parametric data. Categorical variables were compared using the chi-squared test. Univariate correlations were determined using Pearson's coefficient for continuous variables and the point-biserial coefficient for dichotomous variables. Logistic regression analysis of the various clinical and electroanatomic variables was performed to determine the predictors of recurrent AF after catheter ablation. The variables used in multivariate analysis were those with $\mathrm{P}<0.1$ in the univariate models. For predicting the occurrence of PAF in patients aged $\leq 50$ years old and predicting post-RF catheter ablation recurrent $\mathrm{AF}$, area under the receiver operating characteristic (ROC) curve (AUC) was calculated, to determine sensitivity and specificity. The ROC curve was used to calculate the best LTL threshold for predicting PAF in patients aged $\leq 50$ years old and for predicting recurrent $A F$ after RF catheter ablation. The optimal value was defined as that producing the highest sensitivity and specificity to distinguish between different patient groups. $\mathrm{P}<0.05$ was considered statistically significant.

\section{Results}

\section{LTL and PAF}

Baseline characteristics of the healthy individuals and PAF patients are listed in in Table. A total of 105 PAF patients who underwent RF catheter ablation with a mean age of $54.1 \pm 10.8$ years old were enrolled.

Overall, the PAF patients had a shorter LTL than healthy individuals. There was no significant difference in 
A

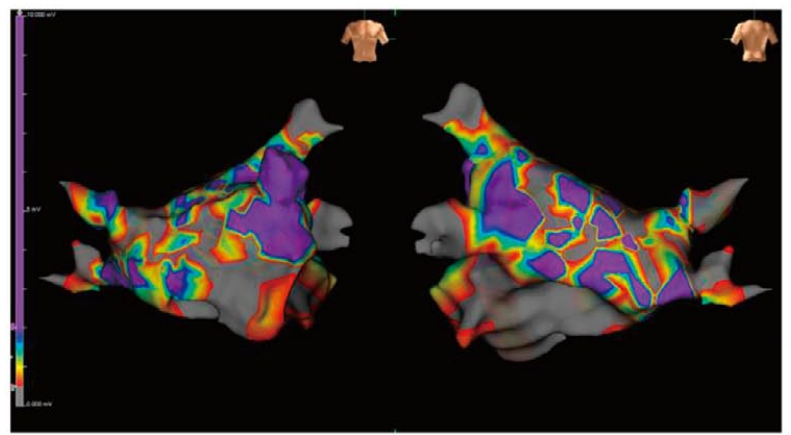

B

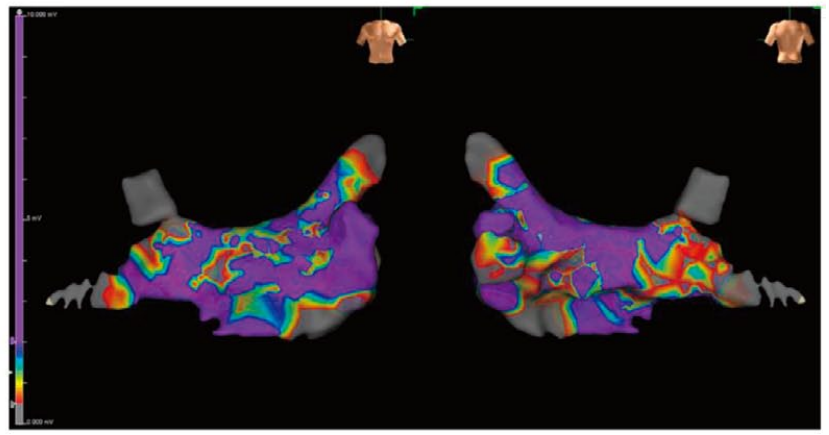

Figure 1. Left atrial (LA) voltage map in anteroposterior view and posteroanterior view. (A) Shorter leukocyte telomere length (LTL) with larger LA low-voltage zone in patients with recurrent atrial fibrillation (AF) after ablation and (B) longer LTL with smaller LA low-voltage zone in patients without post-ablation recurrent AF. Gray, scar zones ( $\leq 0.05 \mathrm{mV})$; purple, normal voltage zones ( $\geq 2 \mathrm{mV})$.
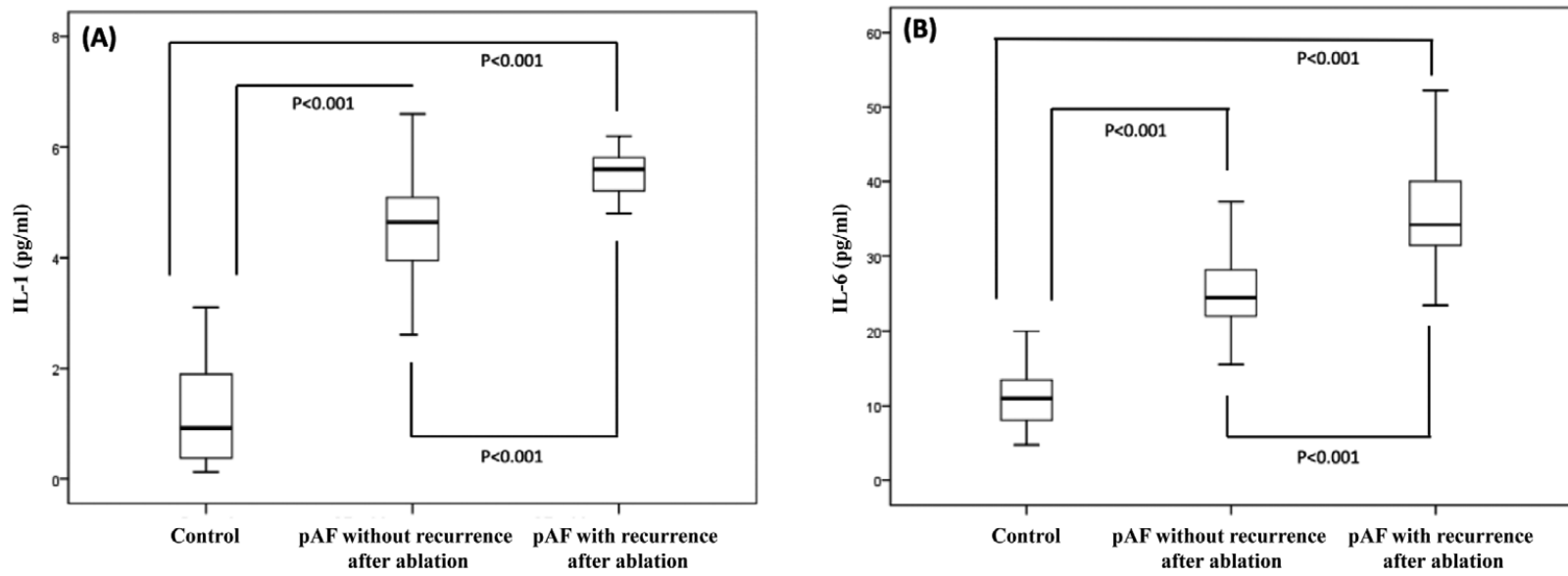

Figure 2. (A) Interleukin (IL)-1 and (B) IL-6 according to presence of paroxysmal atrial fibrillation (PAF) and post-ablation recurrence. Boxes, 25th-75th quartiles; horizontal line, median; Whiskers, the max value and the min value.

LTL between PAF patients and normal subjects at age $>50$ years old $(6.42 \pm 0.82 \mathrm{kbp}$ vs. $6.90 \pm 0.28 \mathrm{kbp}, \mathrm{P}=0.09)$. At $<50$ years old, however, healthy individuals $(n=17)$ had a longer LTL than that in PAF patients $(n=31 ; 7.34 \pm 0.58 \mathrm{kbp}$ vs. $6.44 \pm 0.91 \mathrm{kbp}, \mathrm{P}=0.01$ ). In the present study, $11.4 \%$ of PAF patients $(12 / 105)$ had a non-PV origin, and there was no relationship between origin of AF and LTL in the younger patients, older patients, or the total patient group. In young PAF patients (age $\leq 50$ years), the LTL had a positive correlation with $\mathrm{LA}$ voltage $(\mathrm{R}=0.57, \mathrm{P}=0.001)$ and negative correlation with biatrial scar area $(\mathrm{R}=-0.67, \mathrm{P}<0.001)$.

In the PAF group, there was no significant difference in LTL between patients $<50$ years old and $>50$ years old $(6.44 \pm 0.91 \mathrm{kbp}$ vs. $6.42 \pm 0.82 \mathrm{kbp}, \mathrm{P}=0.92)$. There was also no significant correlation between LTL and other clinical characteristics, such as gender, hypertension, DM, LA diameter or LV ejection fraction (LVEF) in all PAF patients, PAF patients $<50$ years old or $>50$ years old. On ROC curve analysis, LTL $<7.12 \mathrm{kbp}$ could discriminate PAF from nor- mal subjects at age $<50$ years old with a sensitivity of $71 \%$, specificity of $84 \%$, and AUC 0.81 .

\section{Clinical Characteristics vs. Presence of Post-Catheter Ablation AF Recurrence}

A total of 25 patients had recurrent AF after RF catheter ablation. The recurrence rate was $24 \%$ during 1 -year follow-up. The clinical characteristics of patients with and without AF recurrence after RF catheter ablation are listed in Table. There were no significant differences between the patients with and without AF recurrence with regard to age, gender, BMI, BSA, serum creatinine and history of DM, hypertension, or hypercholesterolemia. To investigate the impact of gender on recurrence, we compared LTL between genders and found no significant difference in LTL between male and female patients $(5.71 \pm 0.82 \mathrm{kbp}$ vs. $5.63 \pm 0.87 \mathrm{kbp}$, respectively; $\mathrm{P}=0.82)$ in those with postablation recurrence of $\mathrm{AF}$. 


\section{LTL, Inflammation Markers and Atrial Electroanatomic Remodeling}

The LTL and atrial electroanatomic remodeling parameters are also summarized in Table. Patients with recurrent AF had a significantly shorter LTL $(5.68 \pm 0.82 \mathrm{kbp}$ vs. $6.66 \pm$ $0.71 \mathrm{kbp}, \mathrm{P}<0.001)$, lower LA bipolar voltage $(1.66 \pm 0.17 \mathrm{mV}$ vs. $1.90 \pm 0.22 \mathrm{mV}, \mathrm{P}<0.001)$, larger biatrial scar area index $(3.76 \pm 1.18 \%$ vs. $2.55 \pm 0.62 \%, \mathrm{P}<0.001)$ and larger $\mathrm{LA}$ diameter $(39 \pm 5.38 \mathrm{~mm}$ vs. $34.94 \pm 5.36 \mathrm{~mm}, \mathrm{P}=0.001)$ than those without recurrent AF (Figure 1). Patients with recurrent AF also had significantly higher inflammation markers, that is, IL-1 and IL-6, than those without recurrent AF $(5.62 \pm 0.58 \mathrm{pg} / \mathrm{mL}$ vs. $4.55 \pm 0.79 \mathrm{pg} / \mathrm{mL}, \mathrm{P}<0.001 ; 36.87 \pm$ $11.42 \mathrm{pg} / \mathrm{mL}$ vs. $25.85 \pm 6.69 \mathrm{pg} / \mathrm{mL}, \mathrm{P}<0.001$; respectively; Figure 2). There were no significant differences between those 2 groups with respect to LVEF. LTL was significantly positively associated with LA bipolar voltage $(\mathrm{R}=0.497, \mathrm{P}<0.001)$ and was significantly negatively associated with bi-atrial scar area $(\mathrm{R}=-0.570, \mathrm{P}<0.001)$.

\section{Factors Associated With Recurrent AF}

The predictors of recurrent AF after RF catheter ablation on univariate logistic regression analysis consisted of shorter LTL, lower LA bipolar voltage, larger bi-atrial scar, larger LA diameter and high IL-1 and IL-6 (Table). All of these factors, however, were not independent predictors on multivariate logistic regression analysis. The ROC curves of LTL, LA bipolar voltage and bi-atrial scar area are given in Figure 3. The ROC curve of LTL indicated better power to predict recurrent AF after RF catheter ablation, and it identified patients with post-RF catheter ablation recurrent AF with a specificity of 0.68 and a sensitivity of 0.79 , using a cut-off $<6.14 \mathrm{kbp}$ (AUC, 0.82; 95\% CI: 0.731$0.909, \mathrm{P}<0.001)$.

\section{Discussion}

The major findings of this study are (1) PAF patients had a shorter LTL compared with normal subjects at age $<50$ years old; (2) shorter LTL was associated with a degenerative atrial substrate; and (3) shorter LTL was associated with recurrence of AF after catheter ablation in PAF patients at 1-year follow-up.

\section{LTL and Risk of PAF}

The prevalence of AF is related to age. ${ }^{2}$ No association has been found between LTL and incident AF, and there is no evidence of relative atrial cell telomere shortening in AF in individuals $\geq 65$ years old.$^{20}$ Chronological aging independent of LTL shortening is responsible for the risk of AF, but the impact of LTL on biological aging in younger age groups has not been studied. ${ }^{20}$ Even in the present study, PAF patients $\leq 50$ years of age had significantly shorter LTL than healthy individuals $(6.44 \pm 0.91 \mathrm{kbp}$ vs. $7.34 \pm 0.58 \mathrm{kbp}$, $\mathrm{P}=0.01$ ), but there was no significant difference between PAF patients $>50$ years of age and healthy subjects $(6.42 \pm 0.82 \mathrm{kbp}$ vs. $6.90 \pm 0.28, \mathrm{P}=0.09)$. Further study is needed to clarify the role of LTL in the older AF population. For the first time, the present study has shown that PAF patients have a shorter LTL compared with normal subjects at age $<50$ years. LTL might play a more important role in the genesis of AF in subjects $<50$ years old due to the presence of fewer confounding factors such as cardiovascular disease, pulmonary disease and some degenerative diseases. Shorter LTL might have a potential to

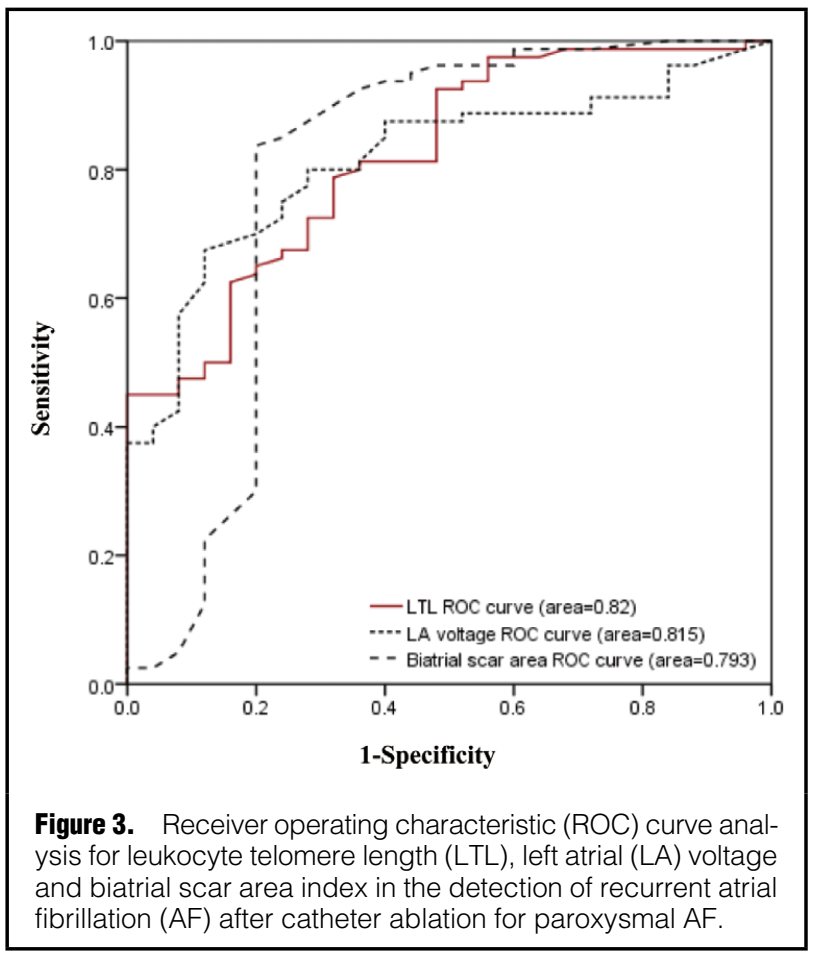

predict PAF in patients aged $<50$ years old, and this finding may be limited to young PAF patients ( $\leq 50$ years), who comprise a small percentage $(<10 \%)$ of all AF patients. Further study is needed to investigate the possible mechanism.

\section{LTL and Atrial Remodeling}

The aging process mediates LA remodeling, including decreased atrial conduction velocity, increased atrial dispersion and disturbed LA local potential characteristics, which may increase the incidence of AF.21-23 Telomeres have been implicated as potential mediators of the biological aging process, and reduced telomere length is associated with increased risk for many age-dependent cardiovascular disease. ${ }^{24}$

Systemic inflammation is associated with AF and its pathogenesis. $^{25}$ IL-1 and IL-6 are systolic inflammation markers. IL-6, in particular, has an effect on the initiation and progression of AF. ${ }^{26}$ The inflammation reaction could accelerate LTL shorting by promoting leukocyte turnover and replicative senescence. ${ }^{27}$ Cumulative inflammatory load, as indexed by high IL-6, is associated with shorter LTL. ${ }^{18}$ Shorter LTL can occur as a result of chronic inflammation reaction, which is correlated with cardiovascular disease. ${ }^{19}$

In the present study, LTL was significantly positively associated with LA bipolar voltage, and was significantly negatively associated with LA diameter and bi-atrial scar area. Shorter LTL was better than chronological age in reflecting chronic inflammatory-related and age-related atrial electric remodeling in young patients with PAF.

\section{LTL Is Associated With Recurrent AF}

RF catheter ablation for all 4 PV isolations with conduction block between the PV and LA is the standard procedure to treat symptomatic PAF and to maintain SR.4,28 In general, successful maintenance of SR at 1 year after PAF 
catheter ablation ranges from $75 \%$ to $93 \%{ }^{29-31}$ In the present study, LA substrate remodeling with a progressive decrease in LA voltage has been shown in patients with recurrent AF after RF catheter ablation. In the present study, we also found a larger scar area and low LA voltage in patients with recurrent AF after RF catheter ablation compared with those without recurrent AF. The aging process is associated with the presence of atrial interstitial fibrosis in animal studies. ${ }^{32,33}$ In a human study, it was also shown that the aging process is associated with the development of excessive collagenous septa that separated small groups of fibers, to produce electrical LA remodeling. ${ }^{22}$ In our previous study, lower LA voltage was associated with higher recurrence after AF ablation in PAF patients aged $>65$ years. ${ }^{6}$ During the aging process, systemic inflammation reactions may cause AF by increasing the production of interstitial fibrosis tissue in the atrium. ${ }^{34,35}$ In the present study, there was no significant difference in chronological age between the patients with and without recurrent AF after RF catheter ablation. The post-catheter ablation recurrent AF patients had shorter LTL and higher IL-1 and IL-6. This indicates that not only chronological age but also the biologic aging process mediates systemic inflammation, which can be a predictor of recurrent AF after PAF ablation.

\section{Clinical Implications}

PAF is sometimes difficult to detect and diagnose on ECG, especially when it is silent and asymptomatic. The present study has shown that LTL $<7.12 \mathrm{kbp}$ can discriminate AF from normal subjects at age $<50$ years old. LTL might have a potential to predict AF in patients aged $<50$ years old. A large, prospective study is warranted to clarify the role of LTL as a biomarker to detect PAF in selected people. Moreover, we pointed out that patients with recurrent AF after catheter ablation had a shorter LTL than those without recurrence. Progressive biological age-related LA electric remodeling including decreased LA voltage and increased bi-atrial scar area may be responsible for the post-RF catheter ablation recurrent AF. Shorter LTL might predict recurrent AF after catheter ablation at 1-year follow-up. Patients with non-recurrence, however, were followed for only 1 year, and therefore may still have recurrence of AF in the long-term follow-up period. Shorter LTL represents the complex aging process and the atrial remodeling process of LA electroanatomic remodeling, which are the confounding factors for post-ablation recurrence of AF. This may explain why LTL is not an independent predictor of recurrent AF after ablation. Nevertheless, on ROC curve analysis, LTL $<6.14 \mathrm{kbp}$ was associated with recurrent AF after catheter ablation.

\section{Conclusion}

PAF patients had a shorter LTL compared with normal subjects at age $\leq 50$ years old. Shorter LTL was associated with a degenerative atrial substrate and post-catheter ablation AF recurrence in patients with PAF.

\section{References}

1. Wolf PA, Mitchell JB, Baker CS, Kannel WB, D'Agostino RB. Impact of atrial fibrillation on mortality, stroke, and medical costs. Arch Intern Med 1998; 158: 229-234.

2. Feinberg WM, Blackshear JL, Laupacis A, Kronmal R, Hart RG. Prevalence, age distribution, and gender of patients with atrial fibrillation: Analysis and implications. Arch Intern Med 1995; 155: 469-473.

3. Psaty BM, Manolio TA, Kuller LH, Kronmal RA, Cushman M, Fried LP, et al. Incidence of and risk factors for atrial fibrillation in older adults. Circulation 1997; 96: 2455-2461.

4. Chen SA, Hsieh MH, Tai CT, Tsai CF, Prakash VS, Yu WC, et al. Initiation of atrial fibrillation by ectopic beats originating from the pulmonary veins: Electrophysiological characteristics, pharmacological responses, and effects of radiofrequency ablation. Circulation 1999; 100: 1879-1886.

5. Chao TF, Cheng CC, Lin WS, Tsao HM, Lin YJ, Chang SL, et al. Associations among the CHADS(2) score, atrial substrate properties, and outcome of catheter ablation in patients with paroxysmal atrial fibrillation. Heart Rhythm 2011; 8: 11551159.

6. Tuan TC, Chang SL, Tsao HM, Tai CT, Lin YJ, Hu YF, et al. The impact of age on the electroanatomical characteristics and outcome of catheter ablation in patients with atrial fibrillation. $J$ Cardiovasc Electrophysiol 2010; 21: 966-972.

7. Chang SL, Tai CT, Lin YJ, Wongcharoen W, Lo LW, Lee KT, et al. Biatrial substrate properties in patients with atrial fibrillation. J Cardiovasc Electrophysiol 2007; 18: 1134-1139.

8. Kistler PM, Sanders P, Fynn SP, Stevenson IH, Spence SJ, Vohra $\mathrm{JK}$, et al. Electrophysiologic and electroanatomic changes in the human atrium associated with age. $J$ Am Coll Cardiol 2004; 44: $109-116$.

9. Issac TT, Dokainish H, Lakkis NM. Role of inflammation in initiation and perpetuation of atrial fibrillation: A systematic review of the published data. J Am Coll Cardiol 2007; 50: 2021 2028.

10. Zhu H, Belcher M, van der Harst P. Healthy aging and disease: Role for telomere biology? Clin Sci (Lond) 2011; 120: 427440.

11. O'Sullivan RJ, Karlseder J. Telomeres: Protecting chromosomes against genome instability. Nat Rev Mol Cell Biol 2010; 11: 171 181.

12. Brouilette SW, Moore JS, McMahon AD, Thompson JR, Ford I, Shepherd J, et al. Telomere length, risk of coronary heart disease, and statin treatment in the West of Scotland Primary Prevention Study: A nested case-control study. Lancet 2007; 369: $107-114$.

13. Samani NJ, Boultby R, Butler R, Thompson JR, Goodall AH. Telomere shortening in atherosclerosis. Lancet 2001; 358: $472-$ 473.

14. van der Harst P, van der Steege G, de Boer RA, Woors AA, Hall AS, Mulder MJ, et al. Telomere length of circulating leukocytes is decreased in patients with chronic heart failure. $\mathrm{J} \mathrm{Am}$ Coll Cardiol 2007; 49: 1459-1464.

15. Vasan RS, Demissie S, Kimura M, Cupples LA, White C, Gardner JP, et al. Association of leukocyte telomere length with echocardiographic left ventricular mass: The Framingham heart study. Circulation 2009; 120: 1195-1202.

16. Chang SL, Tai CT, Lin YJ, Ong MG, Wongcharoen W, Lo LW, et al. The electroanatomic characteristics of the cavotricuspid isthmus: Implications for the catheter ablation of atrial flutter. $J$ Cardiovasc Electrophysiol 2007; 18: 18-22.

17. Chang SL, Tai CT, Lin YJ, Wongcharoen W, Lo LW, Tuan TC, et al. The efficacy of inducibility and circumferential ablation with pulmonary vein isolation in patients with paroxysmal atrial fibrillation. J Cardiovasc Electrophysiol 2007; 18: 607-611.

18. O'Donovan A, Pantell MS, Puterman E, Dhabhar FS, Blackburn $\mathrm{EH}$, Yaffe K, et al. Cumulative inflammatory load is associated with short leukocyte telomere length in the Health, Aging and Body Composition Study. PLoS One 2011; 6: e19687.

19. Rehkopf DH, Needham BL, Lin J, Blackburn EH, Zota AR, Wojcicki JM, et al. Leukocyte telomere length in relation to 17 biomarkers of cardiovascular disease risk: A cross-sectional study of US adults. PLoS Med 2016; 13: e1002188.

20. Roberts JD, Dewland TA, Longoria J, Fitzpatrick AL, Ziv E, $\mathrm{Hu} \mathrm{D}$, et al. Telomere length and the risk of atrial fibrillation: Insights into the role of biological versus chronological aging. Circ Arrhythm Electrophysiol 2014; 7: 1026-1032.

21. Anyukhovsky EP, Sosunov EA, Chandra P, Rosen TS, Boyden PA, Danilo P Jr, et al. Age-associated changes in electrophysiologic remodeling: A potential contributor to initiation of atrial fibrillation. Cardiovasc Res 2005; 66: 353-363.

22. Spach MS, Dolber PC. Relating extracellular potentials and their derivatives to anisotropic propagation at a microscopic level in human cardiac muscle: Evidence for electrical uncoupling of side-to-side fiber connections with increasing age. Circ Res 1986; 
58: $356-371$.

23. Wongcharoen W, Chen YC, Chen YJ, Lin CI, Chen SA. Effects of aging and ouabain on left atrial arrhythmogenicity. J Cardiovasc Electrophysiol 2007; 18: 526-531.

24. Blackburn EH. Switching and signaling at the telomere. Cell 2001; 106: 661-673.

25. Aviles RJ, Martin DO, Apperson-Hansen C, Houghtaling PL, Rautaharju P, Kronmal RA, et al. Inflammation as a risk factor for atrial fibrillation. Circulation 2003; 108: 3006-3010.

26. Cheng T, Wang XF, Hou YT, Zhang L. Correlation between atrial fibrillation, serum amyloid protein $\mathrm{A}$ and other inflammatory cytokines. Mol Med Rep 2012; 6: 581-584.

27. Aviv A. Telomeres and human aging: Facts and fibs. Sci Aging Knowledge Environ 2004; 2004: pe43.

28. Pappone C, Rosanio S, Oreto G, Tocchi M, Gugliotta F, Vicedomini G, et al. Circumferential radiofrequency ablation of pulmonary vein ostia: A new anatomic approach for curing atrial fibrillation. Circulation 2000; 102: 2619-2628.

29. Jais P, Cauchemez B, Macle L, Daoud E, Khairy P, Subbiah R, et al. Catheter ablation versus antiarrhythmic drugs for atrial fibrillation: The A4 study. Circulation 2008; 118: 2498-2505.

30. Oral H, Chugh A, Good E, Sankaran S, Reich SS, Igic P, et al A tailored approach to catheter ablation of paroxysmal atrial fibrillation. Circulation 2006; 113: 1824-1831.

31. Cappato R, Calkins H, Chen SA, Davies W, Iesaka Y, Kalman $\mathrm{J}$, et al. Updated worldwide survey on the methods, efficacy, and safety of catheter ablation for human atrial fibrillation. Circ Arrhythm Electrophysiol 2010; 3: 32-38.

32. Hayashi $\mathrm{H}$, Wang C, Miyauchi Y, Omichi C, Pak HN, Zhou S, et al. Aging-related increase to inducible atrial fibrillation in the rat model. J Cardiovasc Electrophysiol 2002; 13: 801-808.

33. Anyukhovsky EP, Sosunov EA, Plotnikov A, Gainullin RZ, Jhang JS, Marboe CC, et al. Cellular electrophysiologic properties of old canine atria provide a substrate for arrhythmogenesis. Cardiovasc Res 2002; 54: 462-469.

34. Burstein B, Nattel S. Atrial fibrosis: Mechanisms and clinical relevance in atrial fibrillation. J Am Coll Cardiol 2008; 51: $802-$ 809.

35. Boos CJ, Anderson RA, Lip GY. Is atrial fibrillation an inflammatory disorder? Eur Heart J 2006; 27: 136-149. 